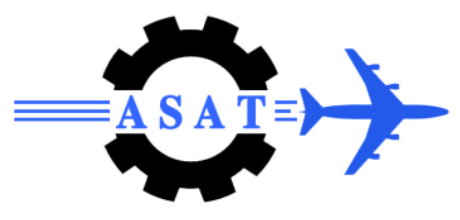

\title{
Aerodynamic Characteristics of a Single and Two Side-By-Side Vertical Circular Cylinders Located in the Incompressible Flow Field
}

\author{
M. H. Gobran*
}

\begin{abstract}
In the present paper, the aerodynamic characteristics of a single vertical cylinder as well as two vertical circular vertical cylinders aligned side-by-side (whose centerline is perpendicular to the flow), located in the incompressible flow field, are numerically investigated. The flow simulations were carried out using unsteady 2D (infinitely long cylinders) and 3D numerical model (finite cylinders) and the finite volume CFD code FLUENT V 6.2.16. The standard $\mathrm{k}-\varepsilon$ turbulence model was used to account for turbulence effects in the flow field but with a special treatment at walls to account for flow separation. In the two dimensional flow fields, two cases were studied: a single cylinder model, where the effect of Reynolds number on the flow characteristics was investigated, and two side-by-side cylinders, where the effect of gap ratio (center-to-center distance divided by the cylinder diameter) is studied. The three dimensional case comprises two models. One model is a single finite cylinder, where the effects of turbulent boundary layer with different velocity gradient $(\delta / \mathrm{H}=0,0.5,0.8$ and 1.3$)$ and the cylinder aspect ratio $(\mathrm{H} / \mathrm{D}=0.5,1$ and 2$)$ are investigated. The other 3-D model consists of two finite cylinders $(\mathrm{H} / \mathrm{D}=0.5)$ in side-by-side arrangement, with different gap ratios ( $\mathrm{L} / \mathrm{D}=1.25,1.5,1.75$ and 2$)$.
\end{abstract}

\section{Nomenclature}

$\mathrm{C}_{\mathrm{D}}$

$\mathrm{C}_{\mathrm{Y}}$

$\mathrm{C}_{\mathrm{P}}$

$\mathrm{D}$

$\mathrm{F}_{\mathrm{X}}$

$F_{Y}$

$\mathrm{H}$

L

$\mathrm{P}$

$\mathrm{P}_{\infty}$

$\operatorname{Re}$

St
Drag coefficient $\left(\frac{2 F_{X}}{\rho U_{\infty}^{2} H D}\right)$

Side force coefficient $\left(\frac{2 F_{Y}}{\rho U_{\infty}^{2} H D}\right)$

pressure coefficient $\left(\frac{2\left(P-P_{\infty}\right)}{\rho U_{\infty}^{2}}\right.$

Cylinder diameter $(7 \mathrm{~cm})$

Force in the flow direction (X)

Force in $\mathrm{Y}$ direction

Cylinder height (0.5D, 1D and 2D)

Cylinders center-to-center distance

Static pressure

Free stream velocity

Reynolds number $\left(\frac{f D}{U_{\infty}}\right)$

Strauhal number $\left(\frac{2 F_{X}}{\rho U_{\infty}^{2} H D}\right.$

Mechanical Power Department, Zagazig University, mhgobran@yahoo.com 


$\begin{array}{ll}\mathrm{U}_{\infty} & \text { Free stream velocity } \\ \rho & \text { Flow density } \\ \delta & \text { Boundary layer thickness } \\ \mu & \text { Dynamic viscosity } \\ f & \text { Side force frequency } \\ \theta & \text { Angle in degree } \\ \theta_{\text {sep }} & \text { Separation angle }\end{array}$

\section{1- Introduction}

The aerodynamic characteristics of three dimensional bluff bodies and flow details around them have attracted much attention due to engineering requirements for the construction of high structure or tall buildings as well as the increase in driving speeds of truck/ semi-trailer or high speed trains. Many applications, such as high-rise bluff bodies, tall building, stacks and oil tanks can be idealized as a finite cylinder with one end placed on the ground plane and embedded in an atmospheric boundary layer of several hundred meters.

Another major practical application is to have a better understanding of the flow around a bundle of risers which links the seabed to the offshore platforms used for oil exploration. These risers are subject to shear and oscillatory flows due to currents and waves, respectively, flows with a very high degree of complexity, with changes of intensity and direction the deeper the water depth.

These applications can be simulated by studying the flow past an obstacle immersed in a turbulent boundary layer on the ground plane. Generally, the flow around a finite cylinder is considered to be three dimensional because of the flow non-uniformity, the presence of longitudinal vortices and low aspect ratio (H/D). The free end of the cylinder is a direct and significant factor for flow three-dimensionality.

Okamoto and Yagita [1984] described a flow over different aspect ratio circular cylinder set on a flat plate and immersed in a shear flow with different velocity gradients. The results were compared with that for uniform flow $(\delta / \mathrm{L}=0.01$ to 0.15$)$. They concluded that: the effect of free end is limited to a height of 3D from the cylinder top end, and the local drag coefficient based on the local dynamic pressure increases from the neighborhood of the bottom as H/D and velocity gradient increase.

Kawamura and et al. [1984] studied, experimentally, the effect of three-dimensionality on the flow characteristics around a finite circular cylinder with various aspect ratio $(H / D=1,2,3,4$, $6,8)$. The effect of boundary layer thickness was also examined, using two values of boundary layer thickness $(\delta / \mathrm{H}=0.1$ and 1$)$.

Behr et al. [1994] investigated the effect of lateral boundary locations on the flow characteristics around two-dimensional circular cylinder. The flow is considered to be incompressible with Reynolds number of 100. They concluded that the lateral boundary should be removed by a distance of 8 cylinder diameter.

Michael Breuer [1998] investigated, numerically, the flow characteristics past infinite and finite cylinders (2-D and 3-D) for low sub-critical Reynolds number (3900). He used large eddy simulation (LES) with different discrimination schemes and different number of grids. 
Kareem and Cheng [1999] used discrete cylinder roughness to simulate high Reynolds number flow feature at low Reynolds number flows. The cylinder used was of aspect ratio of 10 and the average inlet Reynolds number of $2.7 \times 10^{4}$. Different wire sizes with different locations on the cylinder surface were combined together with the two different inlet velocity distributions, to deduce six case studies.

Tanaka et al. [1999], investigated time-averaged flow field behind circular cylinder with aspect ratio from 1.25 to 10 placed on a ground plate in a uniform flow at $\mathrm{R}_{\mathrm{e}}=3.7 \times 10^{4}$ and the influences of the aspect ratio on the wake structure have been presented. Three- dimensional velocity distribution was measured from 1 to 20 diameters downstream the cylinder using rotating yaw meter. The effects of the cylinder top shapes on the wake flow have been also examined.

Tsutsui and Igarashi [2000] investigated, experimentally, the air flow a cylinder protuberance, having aspect ratio of 0.35 , mounted on a flat plate. In the cylinder roof, the position of the reattachment point shifts upstream with increase in boundary layer thickness and the separation occurs in the front of the reattachment line. The minimum value of pressure in the laminar boundary layer is higher than that in the turbulent boundary layer. The Position the value of the maximum pressure coefficient is somewhere along the front stagnation line, and vary according to the boundary layer thickness. The minimum pressure coefficient occurs at about $80^{\circ}$ for all values of boundary layer thickness. The local drag coefficient has minimum values near the plane wall and near the upper edge for all values of boundary layer thickness.

Choel and Sang [2000] investigates the free end effects on the flow around a finite cylinder with different aspect ratios of 6, 10 and 13 at Reynolds number of 2000. The pressure distribution around the cylinder was also measured. The main conclusions of this reference are as follows. Near the ground a wavy structure wake is shown. The horseshoe vortex decreases as the cylinder height increases. The approaching flow moves upward, accelerates near the free end and the separates from the cylinder circumference at the free end. In the wake region, behind the cylinder lower portion, the two-vortex shed from the two sides, are not influenced by the downwash flow separated from the finite cylinder free end. For 10 aspect ratio model, the pressure distribution at 0.883 of the cylinder height approach that of the two dimensional one.

Zhifu Gu and Tianfeng Sun [1999], experimentally, investigated the aerodynamic characteristics of the flow around two staggered cylinders. Both have an aspect ratio of 6.4 and the flow properties are measured at mid span.

Meneghini J.R et al. [2001] studied the 2-D flow around two cylinders in side-by-side and tandem arrangements, for a range of Reynolds number from 100 to 200. The effect of the gap between the cylinders on the fluid forces was investigated.

Alam et al. [2003] investigated, experimentally, the flow around two parallel cylinders, located perpendicular to the uniform flow, at Reynolds number of $5.5 \times 10^{4}$ and different gap ratios $\mathrm{L} / \mathrm{D}=1.2$ to 2.2 ).

Park et al. [2003], experimentally, investigate the flow structure around the two side-by-side arrangement finite cylinders. The gap ratio between the two cylinders is varied from 1 to 2 at fixed Reynolds number of $2 \times 10^{4}$. 
Frederich et al. [2008], numerically, simulated the turbulent separated flow around a wallmounted-finite cylinder in order to assess the predictive accuracy of the methods for the unsteady flow field at high Reynolds number. While the results obtained from Large-Eddy Simulation show only minor differences to experiments, the Detached-Eddy Simulation exhibits problems in the attached laminar boundary layer. By neglecting this insuffcient modeling of the transition phenomenon, the Detached-Eddy Simulation performed can be understood as a Large-Eddy Simulation with an alternative subgrid scalemodel that delivers qualitatively comparable results to the Large-Eddy Simulation.

This paper is organized as follows:

1. The 2-D flow around an infinite single cylinder, for a range of Reynolds number $(2.4 \times 103$ to $1.92 \times 105)$, is investigated.

2. The 2-D flow around two infinitely long parallel cylinders for different gap ratio is investigated. The flow is solved at a Reynolds number of 9.6x 104 .

3. The flow around a single finite cylinder immersed on the turbulent boundary layer is investigated. Different boundary layer thicknesses and different aspect ratios are examined. The Reynolds number in all cases has a constant value of $6.74 \times 104$.

4. Finally, the flow characteristics around two finite side-by-side cylinders (each has an aspect ratio $=0.5$ ) immersed in a uniform flow at a Reynolds number of $6.74 \times 104$ is investigated. Different gap ratios between the two cylinders are examined.

\section{2- Numerical Model}

\section{The Mass Conservation Equation}

The equation for conservation of mass in an incompressible flow condition can be written as follows:

$$
\nabla \cdot \mathrm{V}=0
$$

\section{Momentum Conservation Equations}

Conservation of momentum in an inertial reference frame is described by

$$
\rho \frac{\partial \vec{V}}{\partial t}+\rho(\vec{V} \cdot \nabla) \vec{V}=-\nabla P+\nabla \tau
$$

where: $\mathrm{P}$ is the static pressure and, $\tau$ is the stress tensor (described below) and $\vec{V}$ is the velocity vector

$$
\tau=\mu\left[\left(\nabla \vec{V}+\nabla \vec{V}^{T}\right)-\frac{2}{3} \nabla \vec{V} I\right]
$$

where $\mu$ is the molecular viscosity, $\boldsymbol{I}$ is the unit tensor, and the second term on the right hand side is the effect of volume dilation.

\section{Turbulence Modeling}

The standard $\mathrm{k}-\varepsilon$ model used in this simulation is one of the simplest models of turbulence. It is a two-equation model in which the solution of two separate transport equations allows the turbulent velocity and length scales to be independently determined. Robustness, economy, and reasonable accuracy for a wide range of turbulent flows explain its popularity. The standard $\mathrm{k}-\varepsilon$ model is a semi-empirical model based on model transport equations for the turbulence kinetic energy $(\mathrm{k})$ and its dissipation rate $(\varepsilon)$. The model transport equation for $\mathrm{k}$ is derived from the exact equation, while the model transport equation for $\varepsilon$ was obtained using physical reasoning and bears little resemblance to its mathematically exact counterpart. 


\section{3- Studied Cases}

\subsection{Infinitely-long cylinder (single and two)}

The computational domain is illustrated in Fig. (1), which is a rectangle with dimensions of 14Dx42D for single infinite cylinder model and 28Dx42D for two side-by-side infinite cylinders model. For each case the model/models are located at 14D downstream of the inlet velocity. The boundary conditions are set as follows: At the inlet the velocity is introduced where the inlet boundary condition (BC) is called "velocity inlet". At the outlet the condition is unknown and $\mathrm{BC}$ is assumed to be "outlet flow", this is to model a zero diffusion flux for all flow variables. The BC's at the two sides are put to be symmetric, where there is a zero flux for each quantity across these boundaries.

Finally the cylinders surfaces are set to be a solid wall. In the case of two cylinders model the gap ratio (L/D) is varied $(1.25,1.5,1.75,2$, and 3$)$.

These domains are meshed using the Fluent preprocessor, GAMBIT V 2.04. For a single cylinder, the cylinder wall is surrounded by 30 rows of structured boundary layer mesh followed by a structured mesh. The rest of the domain is completed with unstructured quadrilateral mesh. The total number of cells is 13286 , see figure (2-a). For two cylinders, the inlet and outlet boundaries are divided into 100 increments and the upper and lower boundaries are divided into 150 increments. The cylinder wall itself is divided into 100 increments. The cylinders walls are surrounded by 10 rows of a structured boundary layer mesh, to enhance the solution quality, with $0.05 \mathrm{~cm}$ first row height and growth ratio of 1.04 . The rest of the domain is completed with unstructured triangular mesh. The total number of cells is 32243 , see figure (2-b).

\subsection{Finite single cylinder}

Figure (3) shows the general view of the computational domain used in all simulations of the flow around a single three dimensional cylinder with different aspect ratios $(\mathrm{H} / \mathrm{D}=0.5,1$ and 2). This domain is a parallelogram of dimensions 14Dx42Dx7D. This domain is meshed by means of a Cooper scheme, available in GAMBIT V 2.04, in which the bottom face is meshed first then its mesh is used as a source to mesh the entire domain. Mesh used in this bottom face was unstructured quadrilateral mesh, due to difficulty in creating the boundary layer mesh. The three dimensional element is an irregular hexahedron formed by copying the quadrilateral mesh elements of the bottom face in $\mathrm{Z}$ direction domain, see figure (4). The boundary conditions are velocity-inlet, outl-flow for inlet and outlet, respectively. The cylinder surface, as well as the bottom of the domain, is taken as a wall with no slip boundary condition. The remaining sides of the domain are symmetric.

In the case of $H / D=0.5$, turbulent boundary layer flow with $14 \mathrm{~m} / \mathrm{s}$ free stream velocity and different boundary layer thickness $(0.0,0.5,0.8,1.3)$ is introduced to study the effect of boundary layer thickness. For the other cases $(\mathrm{H} / \mathrm{D}=1$ and 2) a uniform flow of $14 \mathrm{~m} / \mathrm{s}$ is introduced.

\subsection{Finite Two Cylinders}

This domain, which has the same boundary conditions as the single finite cylinder (Fig 5), is meshed by means of a Cooper scheme, as mentioned before, see figure 6 . The total number of cells is 350,000 . In this case, the effects of the gap ratio $(\mathrm{L} / \mathrm{D}=1.25,1.5,1.5$ and 2$)$ on both pressure and drag coefficients are studied. A uniform flow with $U_{\infty}=14 \mathrm{~m} / \mathrm{s}\left(R e=6.74 \times 10^{4}\right.$ based on the cylinder diameter) is an inlet velocity input. 


\section{4- Results and Discussion}

\subsection{Single Infinite Cylinder (2-D flow)}

Two dimensional, unsteady viscous flow characteristics around a single infinite cylinder are investigated. Herein, the effects of Reynolds number on the flow characteristics and fluid forces are studied. The Reynolds number is varied from $2.4 \times 10^{3}$ to $1.927 \times 10^{5}$. The mean flow characteristics $\left(C_{P}\right.$ and $\left.C_{D}\right)$ are calculated by averaging them along a single fluctuating lift cycle. Figure (7) gives the variation of mean pressure coefficient around the cylinder surface $(\mathrm{Cp}-\theta)$ for different values of Reynolds number. It is noticed that the value of minimum pressure coefficient decreases as Reynolds number increases, also its location (separation point) moves along the cylinder surface in the flow direction. These results are summarized in figures (8) and (9), respectively. Figures (10) and (11), respectively, illustrate that: as the Reynolds number increases the Strauhal number increases while the drag coefficient decreases.

Figure (12) shows the vortex formation behind the single infinite cylinder during a single lift cycle (it takes 0.0133 seconds).

\subsection{Two Infinite Cylinders}

Herein, the effects of the gap ratio (L/D) on the flow characteristics for Reynolds number of $9.635 \times 10^{4}$ are investigated. Figure (13) shows the variation of mean pressure coefficient around one of the two cylinders (lower cylinder) for different gap ratios. The pressure coefficient distribution becomes symmetrical at L/D of about 3. Figures (14) and (15) show the variation of mean drag and lift coefficients with the gap ratio. As the gap ratio increases both mean drag and lift coefficients decrease until they take their single cylinder values at L/D of about 3. Figure (16) illustrates the location of stagnation point with the variation of the gap ratio. As the gap ratio decreases, the stagnation point moves along the cylinder surface towards the gap. Figure (17) shows the variation of the two separation points along two cylinders walls. As the gap ratio increases, the two points move clockwise along the cylinder wall for the left cylinder and counter clockwise for the right one. The outer separation point (far from the gap) varies from about $80^{\circ}$ to $72^{\circ}$ and the inner one (in the gap side) varies from $80^{\circ}$ to about $93^{\circ}$ when the gap ratio changes from 3 to 1.25 , respectively.

\subsection{Single Finite Cylinder}

Figures (18) through (22) give the flow characteristics for a finite cylinder of 0.5 aspect ratio. A turbulent velocity profile having $14 \mathrm{~m} / \mathrm{s}$ uniform flow $\left(\operatorname{Re}=6.74 \times 10^{4}\right)$ with different boundary layer thickness is applied. Figures (18) and (19) show the pressure coefficient along the cylinder wall at different $\mathrm{Z} / \mathrm{H}$, for two values of boundary layer thickness, $\delta / \mathrm{H}=0$ and 1.26, respectively. Figure (20) shows the variation of pressure coefficient at the cylinder middle span $(\mathrm{Z} / \mathrm{H}=0.5)$ for different boundary layer thickness $(\delta / \mathrm{H}=0,0.5,0.8$ and 1.26). Figure (21) gives the variation of stagnation pressure coefficient with the distance (from the cylinder bottom) along the cylinder height, $\mathrm{Z} / \mathrm{H}$, for different values of boundary layer thickness. It decreases, for all boundary layer thickness values, until $\mathrm{Z} / \mathrm{H}$ of about $15 \%$ then it increases until reaching its maximum value at $\mathrm{Z} / \mathrm{H}$ of about $70 \%$. After that it, steeply, decreases to reach its minimum value at $\mathrm{Z} / \mathrm{H}=100 \%$ (at the cylinder top). It is also obvious that the maximum pressure coefficient decreases with increasing the boundary layer thickness and its location along the cylinder is almost fixed at $\mathrm{Z} / \mathrm{H}=70 \%$. Figure (22) illustrates that the drag coefficient decreases with increasing the boundary layer thickness, it takes a value of about 0.77 times of that produced at uniform flow. From the above figures (18 through 22) we can notice that: for all values of boundary thickness the separation point occurs at about $80^{\circ}$ 
and the boundary layer growth reduces the maximum pressure coefficient and increases the minimum pressure coefficient, resulting in a reduction in the drag coefficient.

Figures (23) and (24) show the variation of pressure coefficient along the cylinder surface for a uniform flow $\left(\operatorname{Re}=6.74 \times 10^{4}\right)$ and aspect ratios of 1 and 2, respectively. Figure (25) gives the variation of pressure coefficient for the uniform flow at mid cylinder span and for different aspect ratio $(\mathrm{H} / \mathrm{D}=0.5,1$ and 2$)$ compared with that for infinite cylinder. It is clear that the minimum pressure coefficient decreases and the maximum one increases as the aspect ratio increases, also the location of separation point angle increases in the flow direction as the aspect decreases. These results are summarized in table (1).

\subsection{Two Finite Cylinders}

Figure (26) gives the pressure coefficient along mid sections of 0.5 aspect ratio cylinder at different gap ratios $(\mathrm{L} / \mathrm{D}=1.25,1.5,1.75$ and 2$)$, while figure (27) gives the variation of the drag coefficient with L/D.

\section{5- Conclusions}

\subsection{Single Infinite Cylinder}

- The minimum mean pressure coefficient decreases as the $\mathbf{R e}$ increases.

- The location of separation point moves downstream along the cylinder wall as the $\mathbf{R e}$ increases, approaching about $80^{\circ}$ at higher ones.

- As Re increases the strauhal number increases while the drag coefficient decreases.

\subsection{Two Infinite Cylinders}

From studying the effects of the $\mathbf{L} / \mathbf{D}$ on the flow parameters we can conclude that:

- As L/D increases both $\mathbf{C}_{\mathbf{D m}}$ and $\mathbf{C}_{\mathrm{ym}}$ decrease, attaining same values as a single infinite cylinder values at $\mathbf{L} / \mathbf{D}$ of about 3 .

- The location of stagnation points move along each cylinder surface away from the gap toward the gap as $\mathbf{L} / \mathbf{D}$ decreases.

- The locations of the two separation points on each cylinder move along its surface in clockwise of the left cylinder and counter clockwise for the right one as the gap ratio increase.

\subsection{Finite Single Cylinder}

For the cylinder of 0.5 aspect ratio and Reynolds number of $6.74 \times 10^{4}$, the effect of boundary layer thickness on the flow characteristics was investigated. Also the effects of aspect ratios were considered $(\mathrm{H} / \mathrm{D}=0.5,1,2$, and $\infty$, where $\infty$ means infinitely long cylinder or two dimensional case ). The main conclusions are:

- At mean cylinder length (for an example), the minimum pressure coefficient increases and the maximum pressure coefficient decreases as the boundary layer thickness increases.

- The drag coefficient decreases as the boundary layer thickness increases.

- As the aspect ratio increases the minimum pressure coefficient decreases while the maximum one increases.

\subsection{Two Staggered Finite Cylinders}

The main conclusion is that: the drag coefficient decreases with increasing the gap ratio. This is same for two infinite cylinders. 


\section{Acknowledgment}

The author would like to acknowledge Prof. Dr. Mohamed Rafat Shalaan and Prof. Dr Ahmed Fayez Abd-el-Azim, in mechanical power department, Zagazig University for their invaluable contributions to this work.

\section{References}

[1] Alam Md, Moriya M, and Sakamoto H.,"Aerodynamic characteristics of two side-byside circular cylinders and application of wavelet analysis on the switching phenomenon", Journal of Fluids and Structures 18 (2003)325 -346.

[2] Behr M., Hastreiter D. and Tezduyar T.E., "Incompressible flow past a circular cylinder: dependence of the computed flow field on the location on the lateral boundaries", AEM/AHPCRC, Super computer institute, University of Minnesota, July 26, 1994

[3] Cheol-Woo Park and Sang-Joon Lee "Free end effect on the near wake flow structure behind a finite circular cylinder", Journal of Wind Engineering and Industrial Aerodynamic 88 (2000) 231-246

[4] Frederich, O, Wassen E \& Thiele F "Prediction of the Flow Around a Short WallMounted Finite Cylinder using LES and DES" Journal of Numerical Analysis, Industrial and Applied Mathematics (JNAIAM) vol. 3, no. 3-4, 2008, pp. 231-247ISSN $1790\{8140$

[5] Kareem A. and Cheng C.M, "Pressure and force fluctuations on isolated roughened circular cylinders of finite height in boundary layer flow", Journal of fluid and structure (1999) 13, 907-933.

[6] Kawamura Takao, Hiwada Munehiko, Hibino Toshihaura and Kumara Malaya, "Flow around circular cylinder on a flat plate", Bulletin of JSME, Vol.27, No.232, October 1984.

[7] Meneghini J.R, Saltara F, Siqueira C.L.R and Ferrari J.A, (Numerical simulation of interference between two circular cylinder in tandem and side-by-side arrangements", Journal of fluid and structure (2001) 15, 327-350.

[8] Michel Breuer, "Numerical and modeling influences on large eddy simulation for the flow past a circular cylinder", International Journal of Heat and fluid flow 19 (1998) 512-521.

[9] Okamoto Shiki and Yagita Miki, "Flow past circular cylinder of finite length placed normal to ground plane in uniform shear flow", Bulletin of JSME, Vol.27, No.229, July 1984.

[10] Park C.W and Lee S.J, "Flow structure around two finite circular cylinders located in an atmospheric boundary layer: side-by-side arrangement", Journal of fluid and structure 17 (2003) 1043-1058.

[11] Tanaka Shuji and Murata Susumu, "An investigation of the wake structure and aerodynamic characteristics of a finite circular cylinder", JSME International Journal, series B, Vol.42, No.2, 1999

[12] Tsutusi Takayuki, Igarashi Tamostu and Nakamura Hajime, "Fluid flow and heat transfer around a cylindrical protuberance mounted on a flat plate boundary layer", JSME International Journal, series B, Vol.43, No.2, 2000

[13] Zhifu Gu and Tianfeng Sun," On interference between two circular cylinders in staggered arrangement at high sub-critical Reynolds numbers", Journal of Wind Engineering and Industrial Aerodynamic 80 (1999) 287-309. 


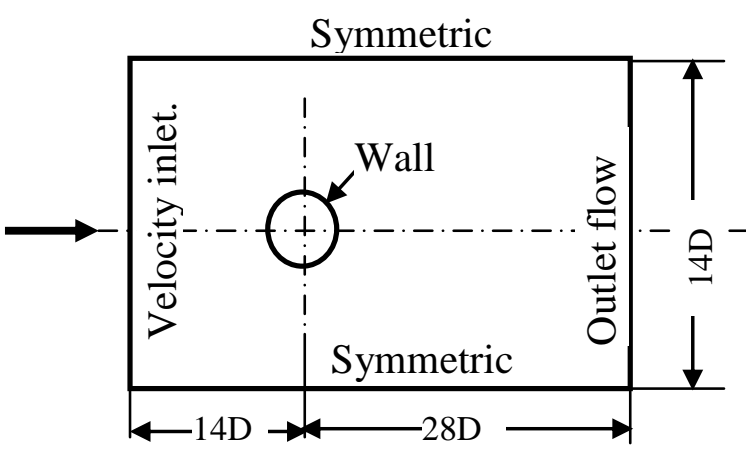

(a)

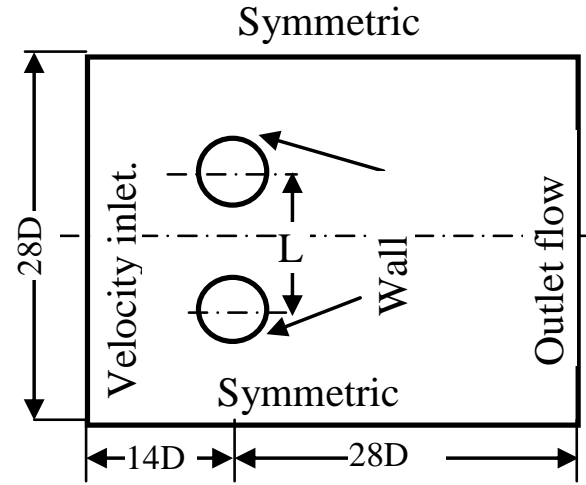

(b)

Fig (1) Computational domain and boundary conditions for 2-D cylinders, (a) single $\quad$ (b) two parallel cylinders

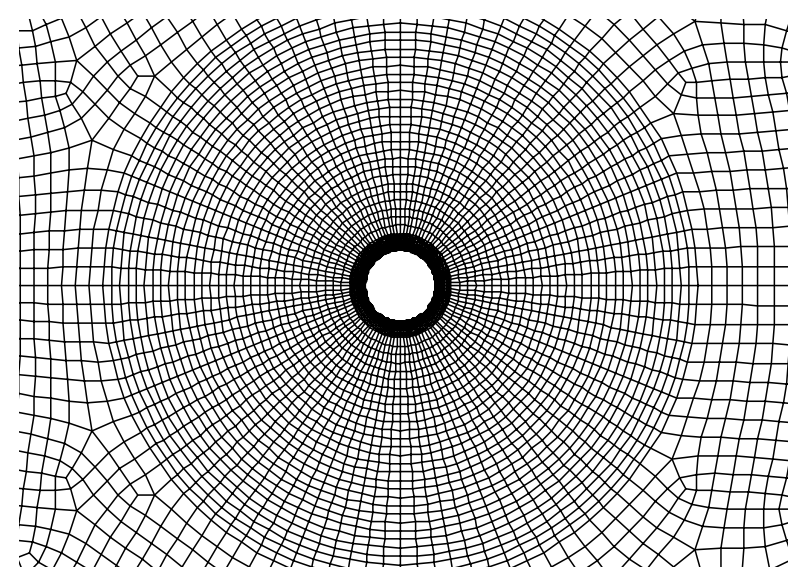

(a)

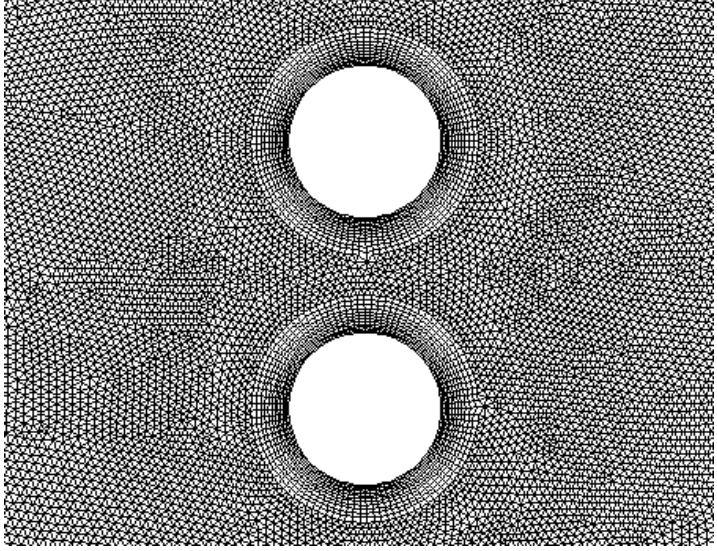

(b)

Fig. (2) Computational grid for: (a) infinite cylinder (b) two staggered infinite cylinder

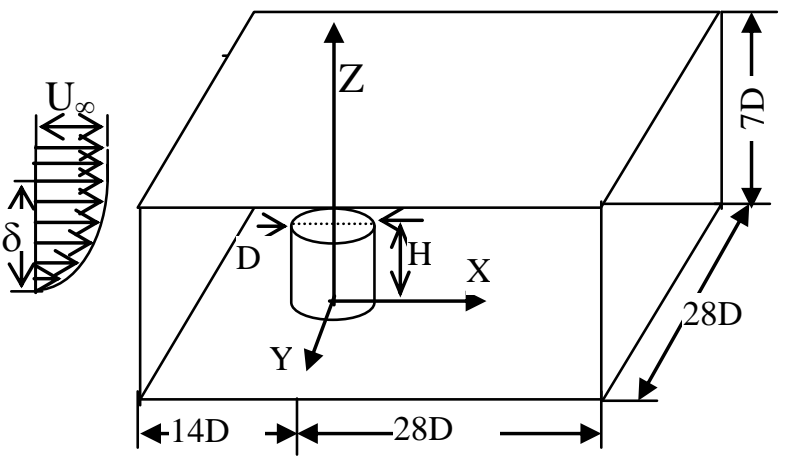

Fig.(3) Computational domain for finite single cylinder

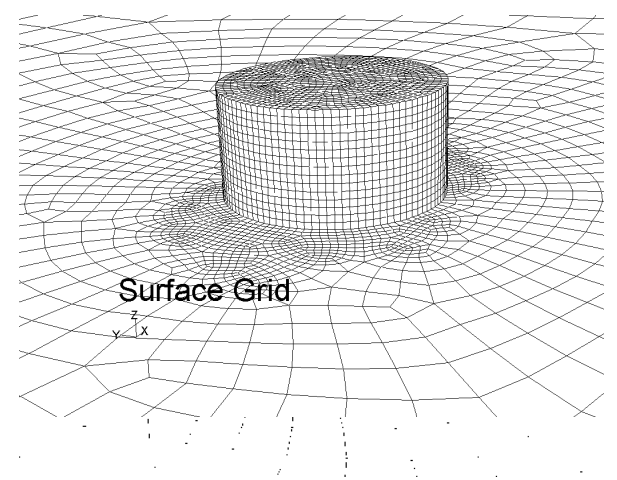

Fig.(4) Surface grid for single finite cylinder 


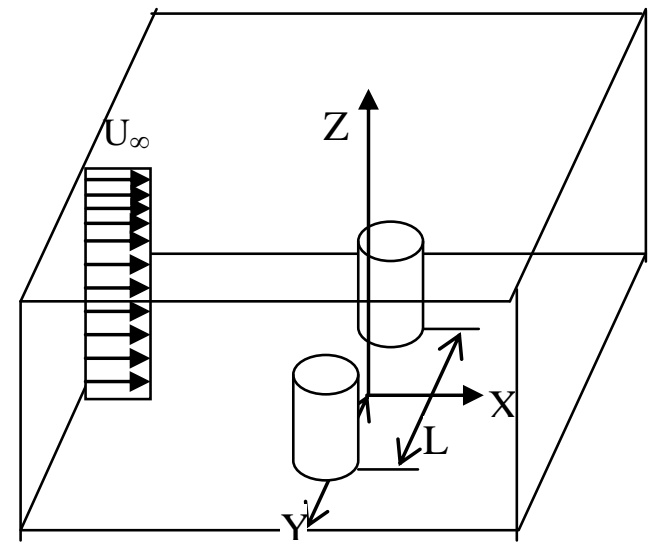

Fig.(5) Computational domain for two finite single cylinders

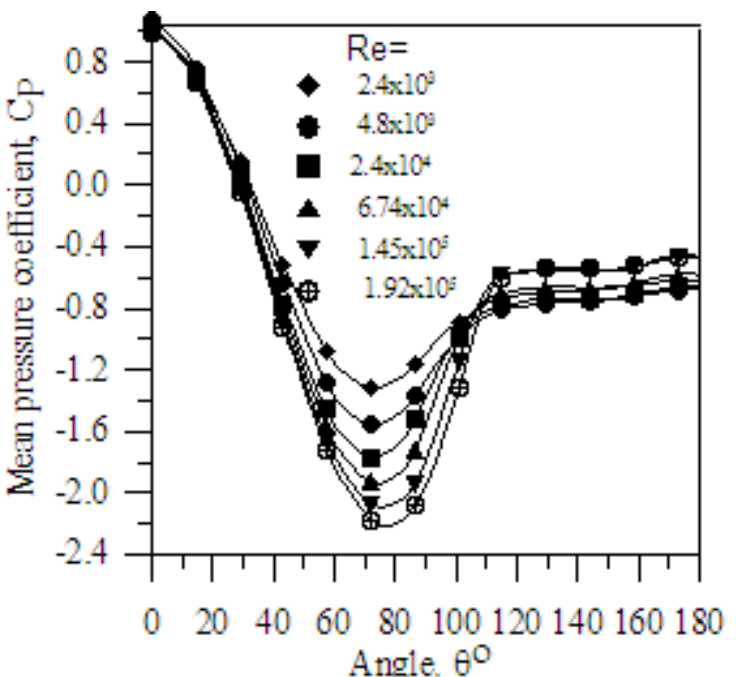

Fig. (7): Mean $C_{p}$ with $\theta$ at different $R e$ for single infinite cylinder

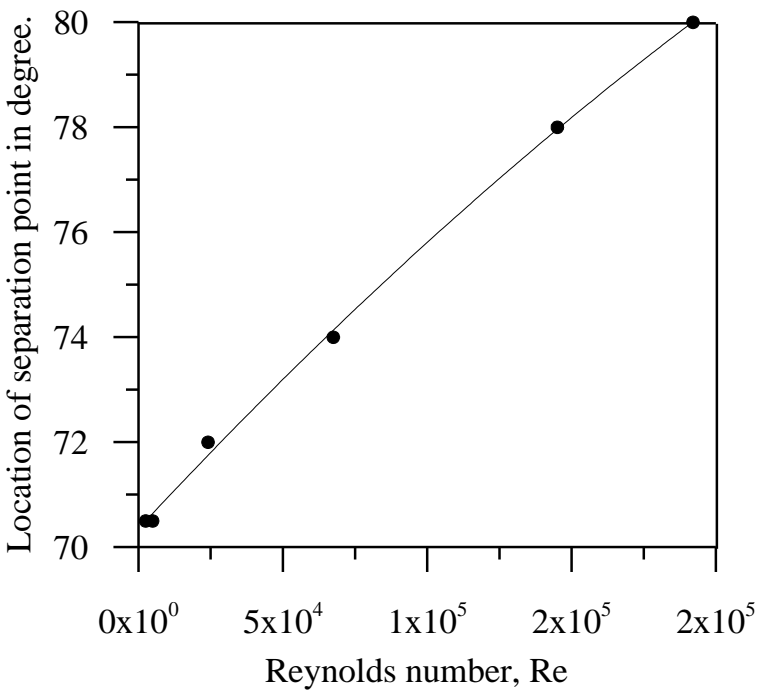

Fig.(9): $\theta_{\text {sep }}$ aginst Re for single infinite cylinder

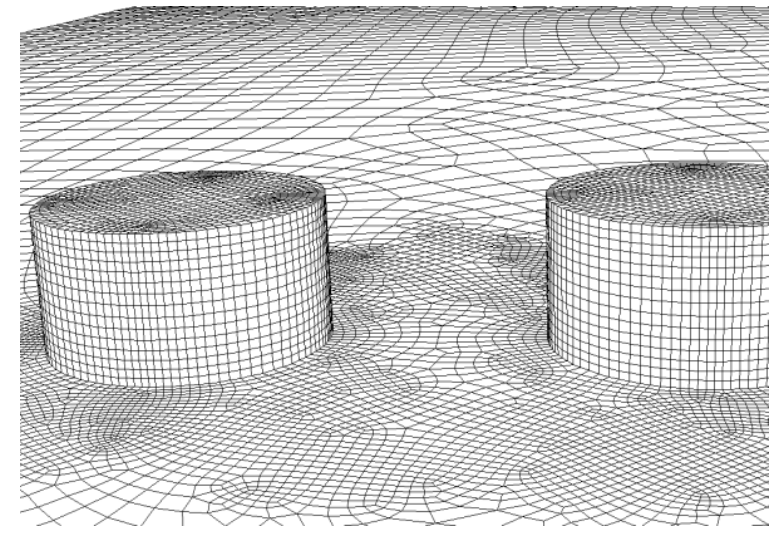

Fig.(6) Surface grid for two finite cylinders

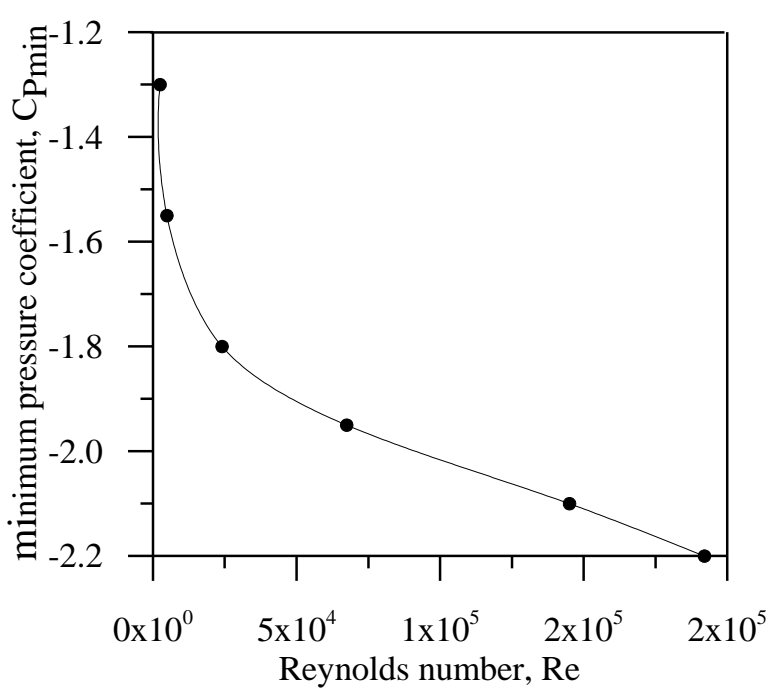

Fig.(8): $C_{P \min }$ against Re for single infinite cylinder

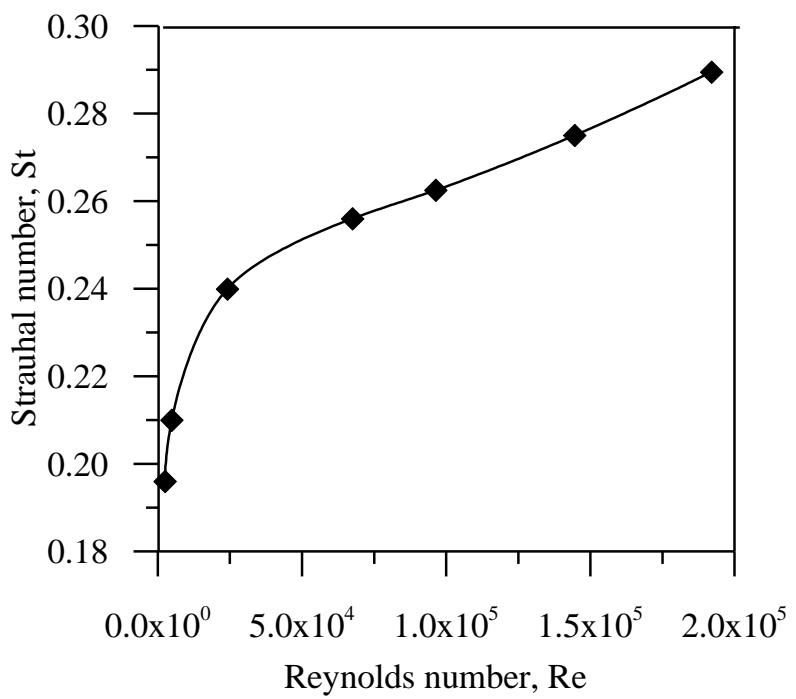

Fig.(10): Strauhal with Re for single infinite cylinder 


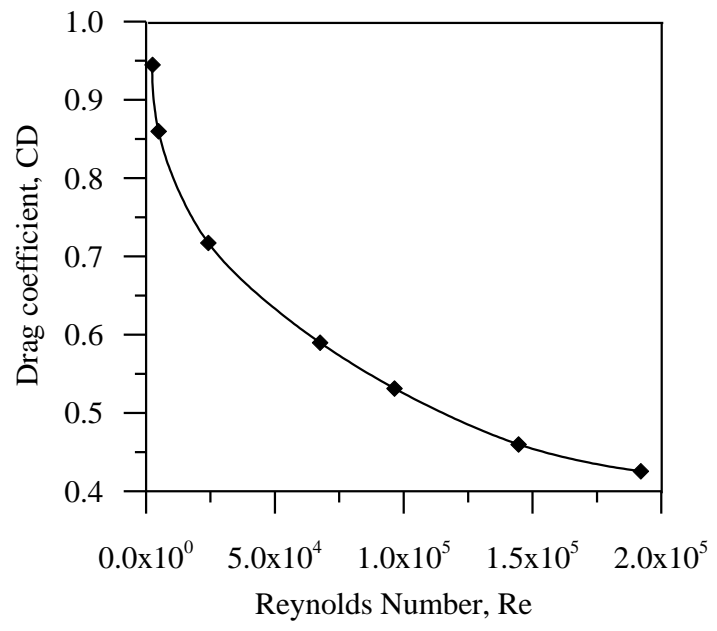

Fig. (11): $C_{D}$ against Re for single infinite cylinder

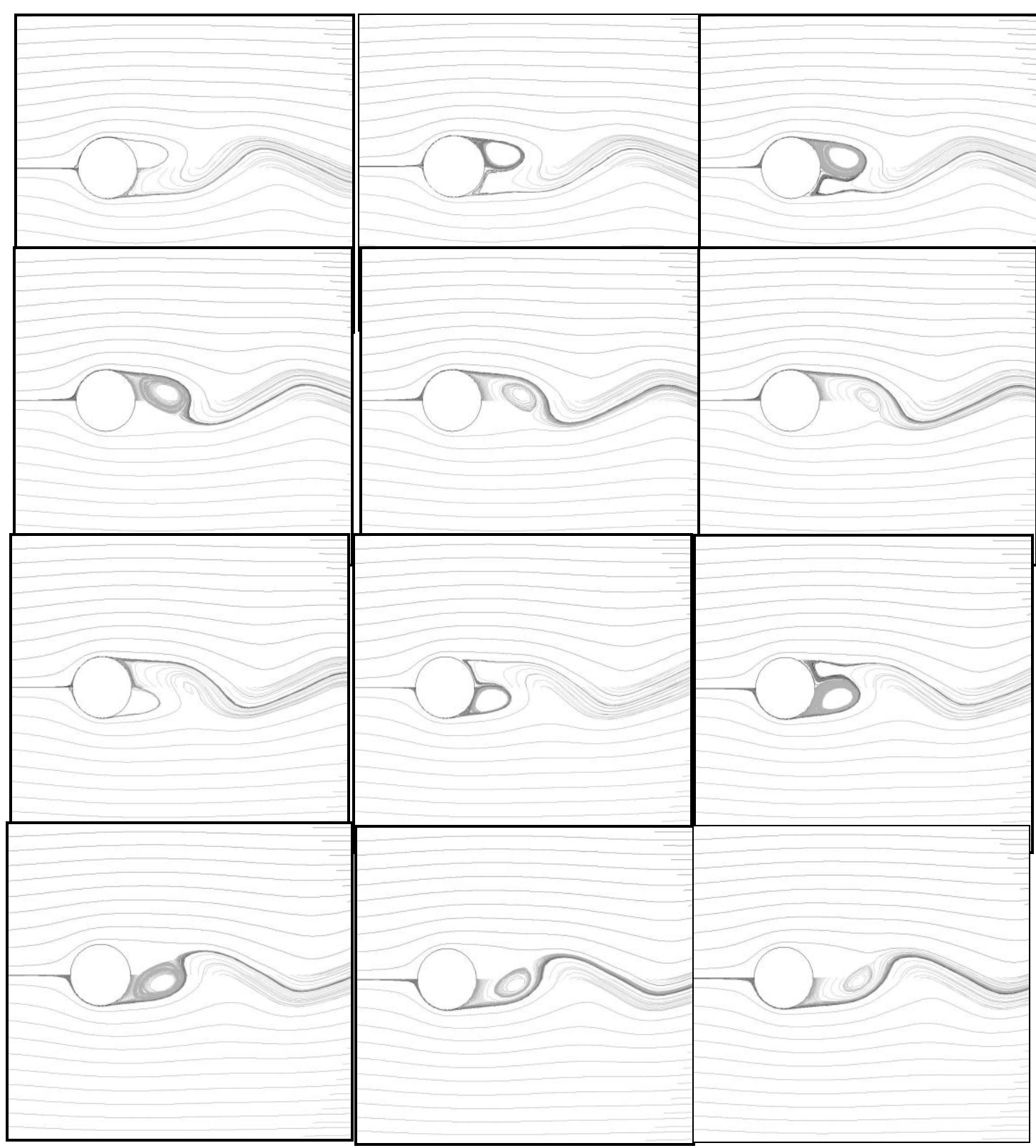

Fig. (12) Vortex formation behind the single infinite cylinder at $\operatorname{Re}=9.635 \times 10^{4}$ during single side force cycle (time interval of 0.0133 second). 


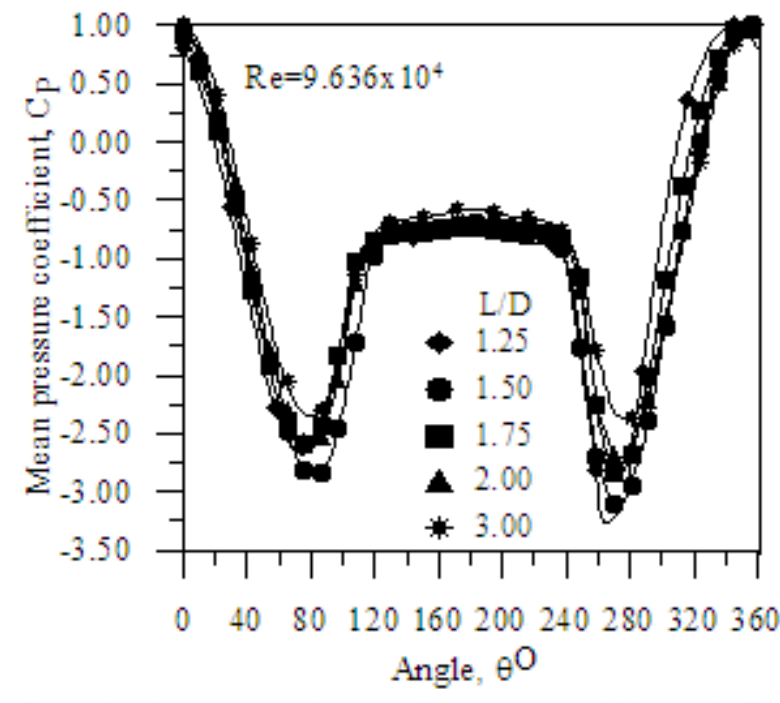

Fig.(13): Variation of $m$ ean $C_{p}$ with $\theta$ at different $L / D$ for one of the two infinite cylindes case

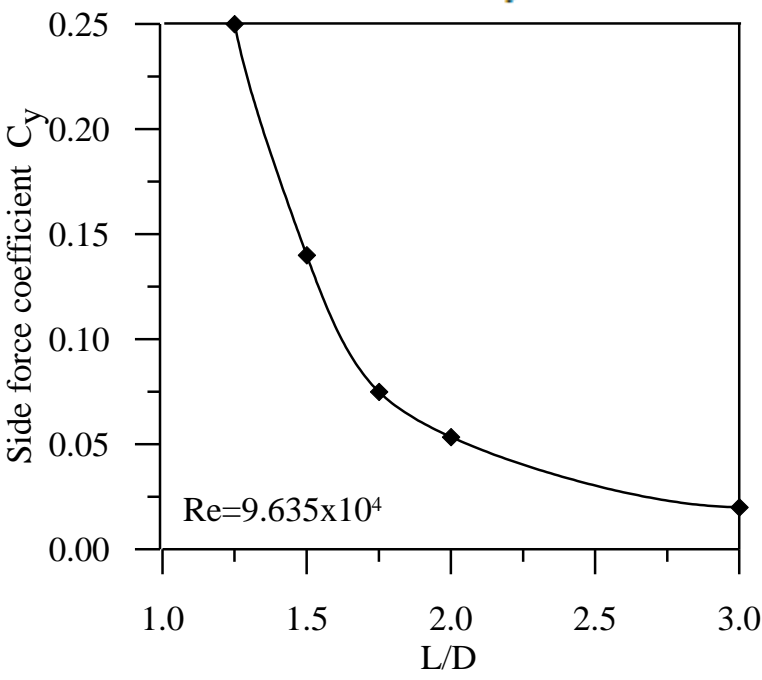

Fig. (15): Side force coefficiuent against L/D on one of the two finite cylinders

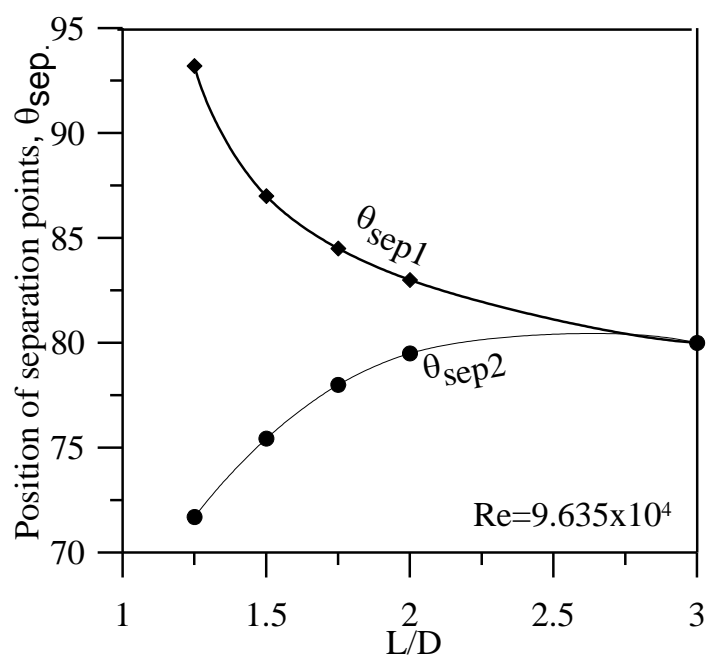

Fig.(17): Separation points variation with L/D for two infinite cylinders

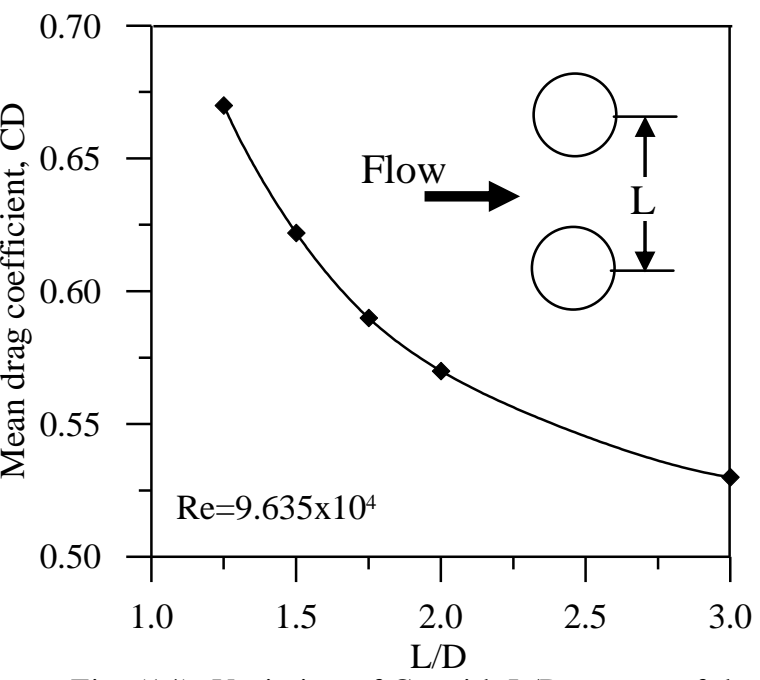

Fig. (14): Variation of $C_{D}$ with $L / D$ on one of the two infinite cylinders

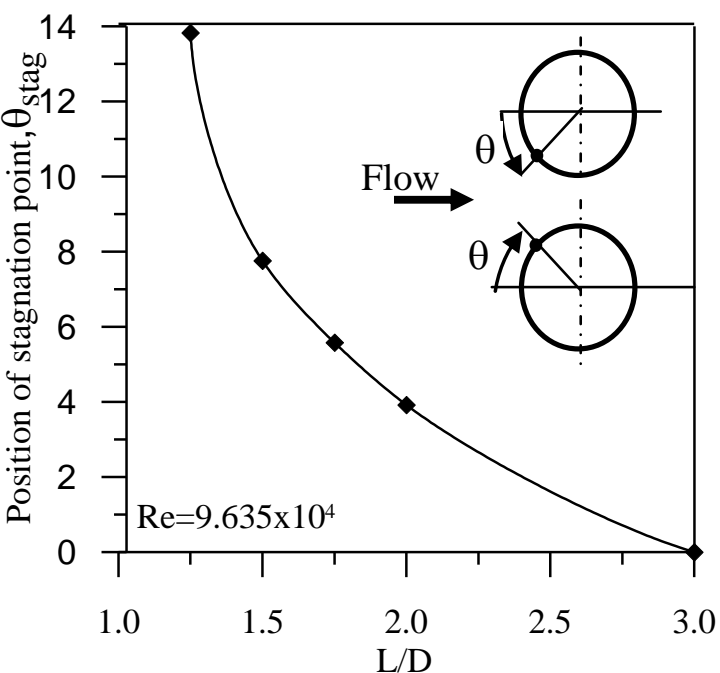

Fig.(16): Stagnation points variation with $\mathrm{L} / \mathrm{D}$ on two infinite cylinders

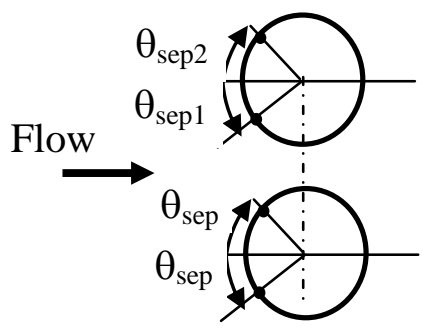



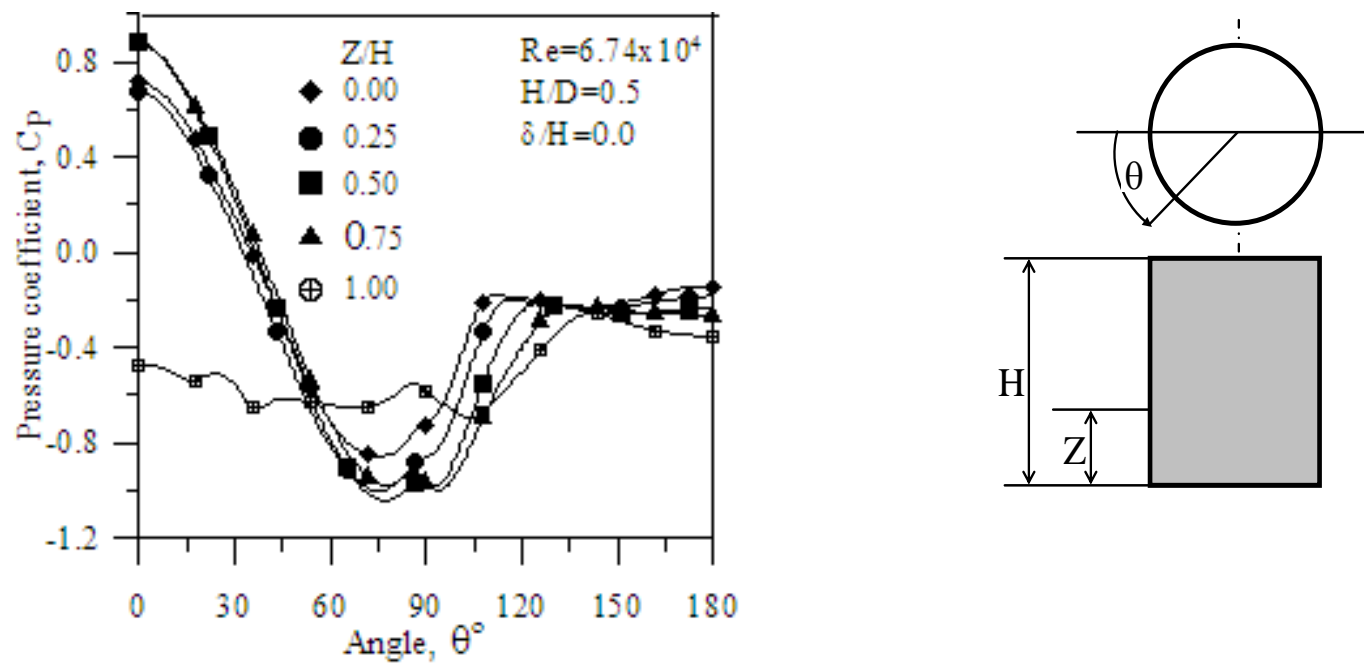

Fig.(18): $C_{p}$ distribution around finite single cylinder

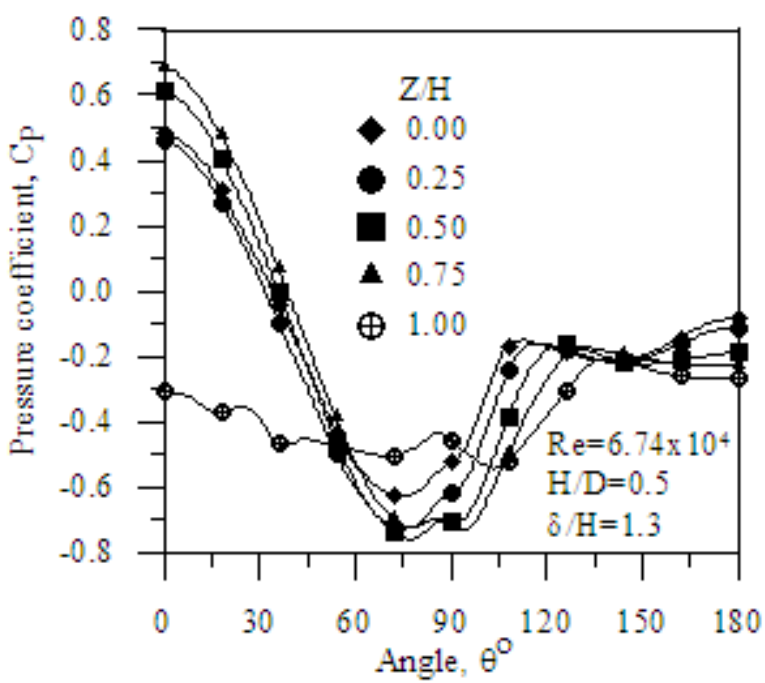

Fig.(19): $C_{p}$ distribution around finite single cylinder

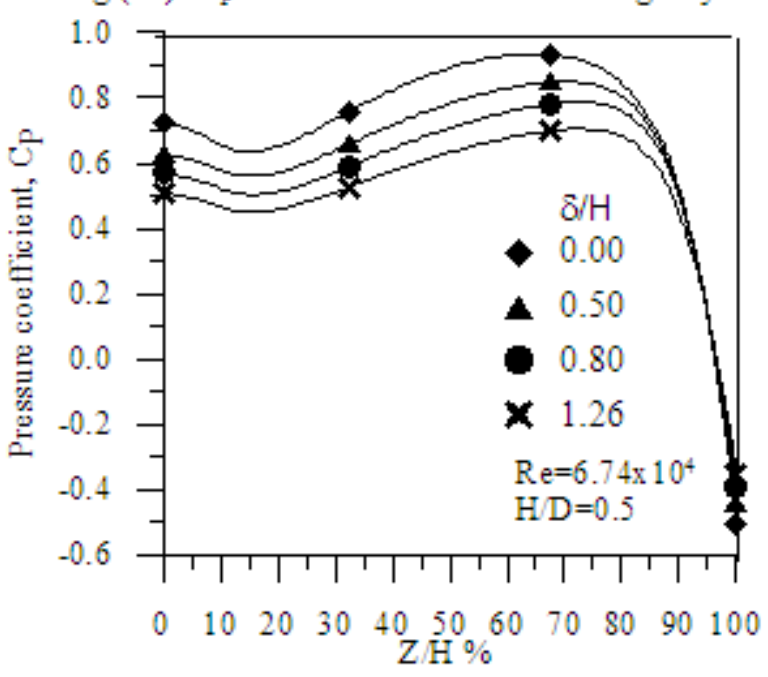

Fig.(21): Variation of $C_{P}$ along front stagnation line of single finite cylinder

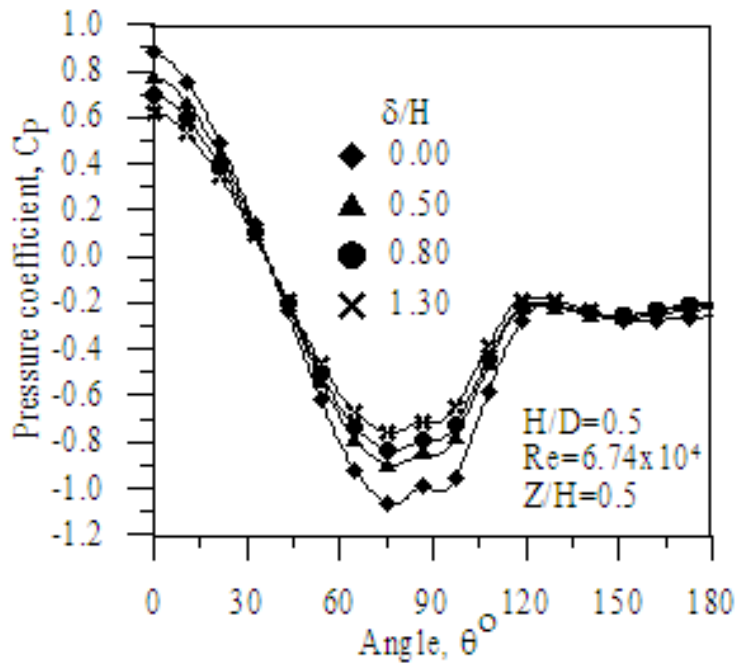

Fig. (20): $C_{p}$ ag ainst $\theta$ for finite single cylinder case at different boundary layer thickness ratio

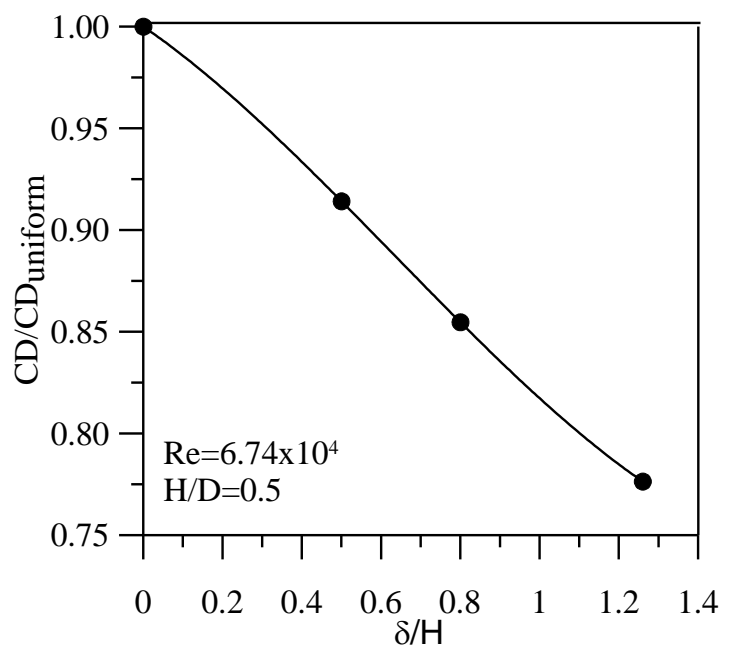

Fig.(22): Variation of $C_{D}$ with $\delta / H$ 


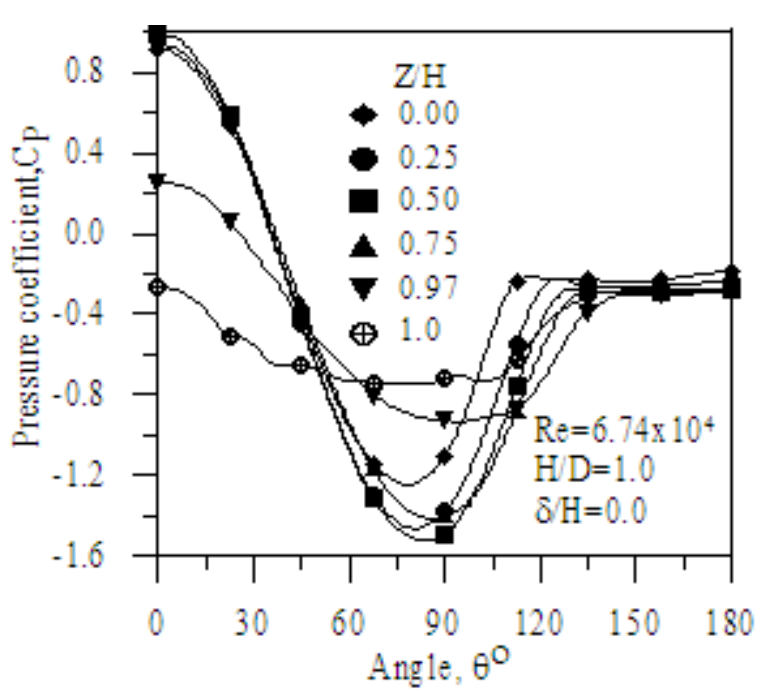

Fig.(23): $C_{p}$ distribution around finite single cylinder

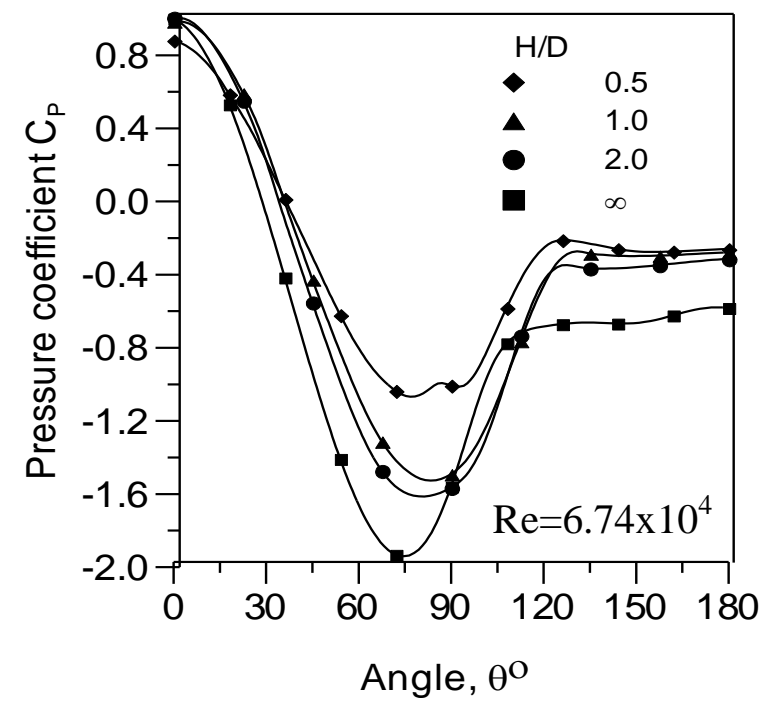

Fig (25) Finite cylinder mean radius pressure coefficient distribution

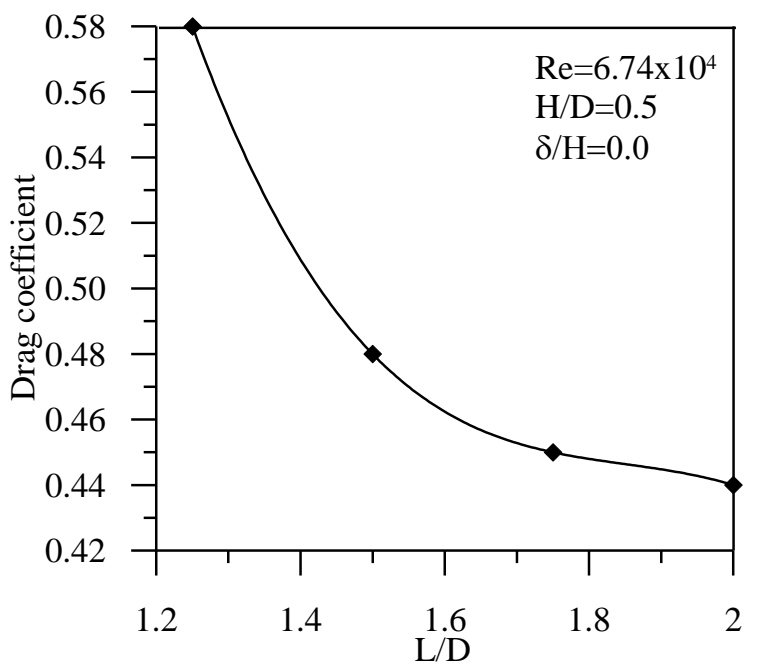

Fig.(27): $C_{D}$ on one of the two finite cylinder with $L / D$

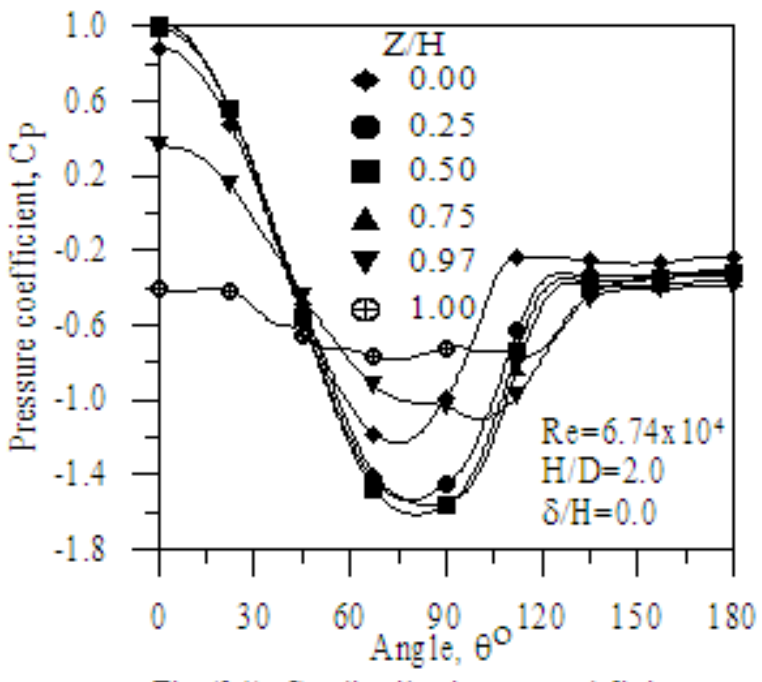

Fig.(24): $C_{P}$ distribution around finite single cylinder

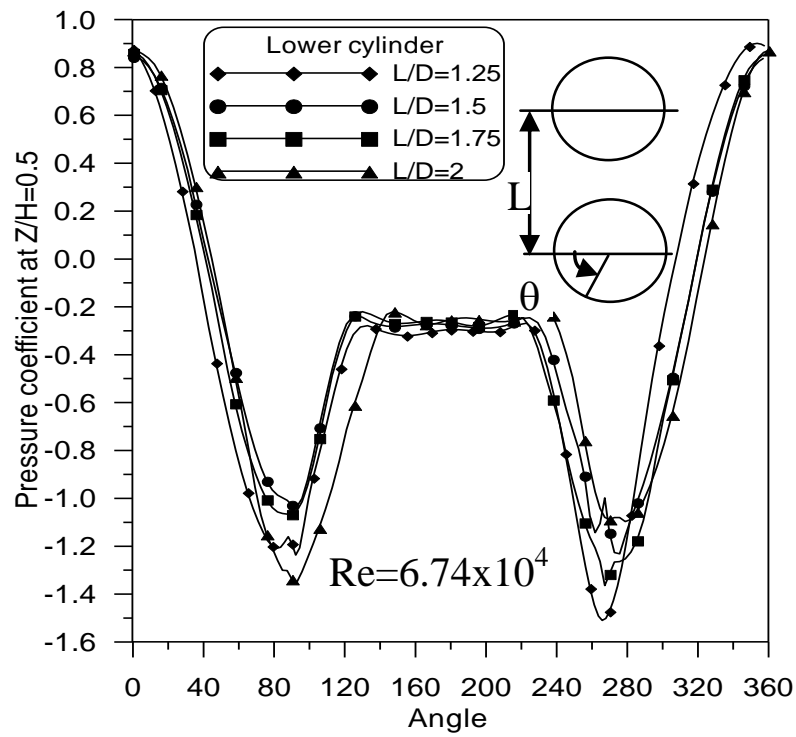

Fig (26) Pressure coefficient at middle cylinder height for two finite cylinders at different gap ratios

Table (1) $\mathbf{C}_{\text {Pmin }}, \mathbf{C}_{P \text { max }}$, and $\theta_{\text {sep }}$ at mean height, $\mathrm{Z} / \mathrm{H}=\mathbf{0 . 5}$, for $3-\mathrm{D}$ cylinder at different $H / D$

\begin{tabular}{c|c|c|c|c}
\hline \hline $\mathrm{H} / \mathrm{D}$ & $\infty$ & 2 & 1 & 0.5 \\
\hline \hline $\mathrm{C}_{\text {Pmin }}$ & -1.932 & -1.62 & -1.55 & -1.05 \\
\hline $\mathrm{C}_{\text {Pmax }}$ & 1 & 1 & 0.985 & 0.88 \\
\hline$\theta_{\text {sep }}$ & 72 & 80 & 83 & 91 \\
\hline \hline
\end{tabular}

